\section{Lighting up the sky}

\section{Washington}

After several delays due to technical problems, bad weather and more technical problems, the Combined Release and Radiation Effects Satellite (CRRES) was launched on 24 July by an Atlas-1 rocket from Cape Canaveral, Florida. From a highly elliptical orbit, CRRES will, over the course of a year, release puffs of fluorescent ionized gas at altitudes ranging from 240 to 21,000 miles, allowing scientists on Earth to study the structure and dynamics of the Earth's magnetic field.

The launch itself marked an important change of style for the National Aeronautics and Space Administration (NASA): instead of buying the rocket and launching it, NASA contracted out the task of putting CRRES into orbit to General Dynamics, the manufacturer of the Atlas- 1 . Efforts to create a commercial space business in the United States were begun by the Reagan administration after the Challenger explosion, but the first commercial launch was not until last year, when McDonnell Douglas used one of its Delta rockets to put a British communications satellite into geostationary orbit (see Nature 341, 5; 1989). In the future, NASA intends that scientific satellites too should be launched by commercial arrangement with the various US rocket manufacturers.

The purpose of CRRES is literally to illuminate the geometry of the Earth's magnetic field in the upper atmosphere and near space. On board are 24 canisters, each containing about $10 \mathrm{~kg}$ of barium, calcium, lithium or strontium along with a small charge of boron and titanium, which, once ignited, burns spontaneously to vaporize the metals and create a cloud of ionized atoms.

Fluorescing under the action of ultraviolet radiation from the Sun, the glowing ion clouds will move along magnetic field lines, betraying their structure for the benefit of researchers.

By virtue of its elliptical orbit, CRRES will be able to release tracers into both the ionosphere, the ionized upper portion of the Earth's atmosphere, and the magnetosphere, the region beyond the atmosphere that is occupied by energetic charged particles supplied by the solar wind.

Both the ionosphere and the magnetosphere are regularly disturbed by outbursts of charged particles from solar flares, disrupting radio transmissions around the Earth. These phenomena have indirectly revealed something of the physical characteristics of the ionosphere and magnetosphere, but, by allowing experiments under controlled conditions, CRRES will be able to paint a more exact picture of these regions.

In addition to its payload of chemical bombs, CRRES also carries a number of instruments supplied by the Department of Defense to measure electromagnetic activity and particle densities in the vicinity of the satellite. These devices will be used to help choose the best moment to release the canisters, and to measure the properties of the ion clouds produced.

According to Gene Wescott of the University of Alaska, a principal investigator on several of the CRRES experiments, the ion clouds created in low-altitude releases should be visible to the naked eye for periods of many minutes. Inhabitants of the Caribbean island of Puerto Rico are likely to get the best show: in June and

July next year, low-altitude CRRES experiments will be augmented by simultaneous releases of ion clouds from sounding rockets.

In some of these experiments, a mixture of barium, calcium and strontium will be released; their different masses and ionization energies make their interaction with the ionosphere visibly different in ways that will diagnose the complex properties of the plasma.

Once the ion clouds disperse and dim, it will take months, perhaps years, for the barium and other metals to descend to the surface. And environmentalists need not be alarmed. The tens of kilograms of barium aboard CRRES are far outweighed by the many tons that drop into the Earth's atmosphere every year in the form of micrometeorites.

David Lindley

\title{
Japan's angel tours the sky
}

\section{Tokyo}

ON Saturday, 4 August, Japan's first lunar probe Hiten (named after a Buddhist angel) will flash past the Moon in one of a rapid succession of lunar passes which, if successful, will send the tiny satellite hurtling off on its furthest venture yet into space. The probe will then return in March for some risky 'aerobrake' experiments in the Earth's upper atmosphere.

At $182 \mathrm{~kg}$, Hiten weighs no more than a Japanese Surno wrestler. But by cleverly exploiting the gravitational fields of the Earth, Moon and Sun, engineers at the Institute of Space and Astronautical Science (ISAS) hope to make Hiten perform a complex series of loops around the Earth and Moon without completely expending the probe's very limited stores of fuel.

Launched by a small solid-fuel rocket in January, Hiten was pulled out of an elliptical Earth orbit in March by the gravity of the approaching Moon. As it swung by the Moon, Hiten dropped a mini-satellite about the size of a basketball into lunar orbit before proceeding into a large elliptical orbit around the Earth extending about $750,000 \mathrm{~km}$ into space.

After nearly four months of leisurely Earth orbits, Hiten again passed close by the Moon on $10 \mathrm{July}$ and was pulled into a tighter Earth orbit, with an apogee of $560,000 \mathrm{~km}$, that brings the probe past the Moon every month.

Following three more lunar passes on 4 August, 7 September and 2 October, the Moon will sling the probe off into space, sending it out to a distance of $1,350,000$ $\mathrm{km}$ from Earth on 17 November before the Moon catches it again early next year and brings it back into a tighter Earth orbit.

Another series of close encounters with the Moon will then force Hiten into an orbit that will bring it careering through the Earth's upper atmosphere at a height of only $120 \mathrm{~km}$ in mid-March 1991 . ISAS engineers then hope to use the braking effects of the Earth's upper atmosphere to bring the high-speed Hiten back under control.

Jun Nishimura, director general of ISAS, says that if the probe survives its ride through the atmosphere it may then be placed at one of the zero-gravity 'Lagrange points', where the gravitational forces due to the Earth and the Sun cancel each other. It will be allowed to just "sit for a while", says Nishimura, after which Hiten's last drops of fuel may be used to push it into a lunar orbit alongside the mini-satellite released in March.

The small lunar orbiter has been the only disappointment of an otherwise successful mission. Shortly before the orbiter was released into lunar orbit, it was realised that the telemetry system of the orbiter was not working, so that ISAS engineers could not track it.

ISAS called on astronomers throughout Japan to train their telescopes on the Moon on 18 March, when the lunar orbiter's tiny engine was fired after it had been released by Hiten. A plume from the rocket was indeed photographed with a telescope on Mount Kiso, $200 \mathrm{~km}$ west of Tokyo, and although the lunar orbiter has never been seen or heard of since, ISAS scientists are confident that it successfully achieved lunar orbit.

But it is still uncertain whether Hiten will eventually join the lunar orbiter. Project manager K. T. Uesugi says the aerobrake experiments in the Earth's upper atmosphere next March are "quite risky" and may turn out to be the" end of Hiten's mission".

David Swinbanks 